Received: 6 June 2017

Accepted: 6 October 2017

Published online: 21 November 2017

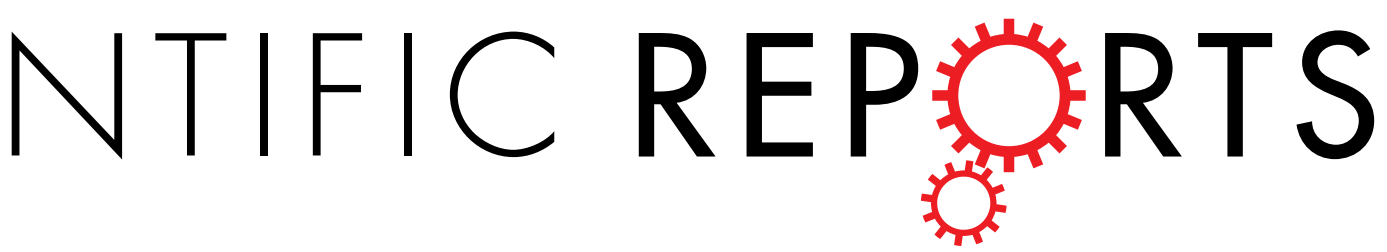

\title{
OPEN Whey Protein Concentrate Renders MDA-MB-231 Cells Sensitive to Rapamycin by Altering Cellular Redox State and Activating GSK3 $\beta$ / mTOR Signaling
}

\author{
Shih-Hsuan Cheng ${ }^{1}$, Yang-Ming Tseng ${ }^{2}$, Szu-Hsien Wu ${ }^{3,4}{ }^{4}$ Shih-Meng Tsai ${ }^{5}$ \& Li-Yu Tsai ${ }^{1}$
}

Whey protein concentrate (WPC) is an amino acid-rich supplement that has been shown to increase cellular antioxidant capacity. Mammalian target of rapamycin (mTOR) is a crucial regulator of signaling in mammalian cells, and serves as a therapeutic target for triple-negative breast cancer (TNBC). This study was designed to investigate the effect of combining WPC with rapamycin on MDA-MB-231 human breast cancer cells. These cells were found to be insensitive to rapamycin and exhibited higher glutathione (GSH) and reactive oxygen species levels than non-tumorigenic MCF-10A cells. However, for MDA-MB-231 cells, the half maximal inhibitory concentration of rapamycin was lower when this drug was administered in combination with WPC than when used alone. Furthermore, combining WPC with rapamycin depleted GSH levels and reduced Nrf2 nuclear accumulation. In addition, WPC activated GSK3 $3 /$ mTOR signaling, and GSK3 3 appeared to be involved in the WPC-mediated Nrf2 reduction and $m$ TOR activation. In conclusion, WPC induced rapamycin sensitivity in MDA-MB- 231 cells by altering their redox state and activating GSK3 $3 /$ mTOR signaling. These results not only suggest a novel therapeutic approach for breast cancer treatment, but also provide insight into the critical pathways affecting the resistance to $m$ TOR inhibition observed in a subgroup of TNBC patients.

Breast cancer is the most common malignancy affecting women worldwide and its incidence has increased globally over recent decades. Triple-negative breast cancer (TNBC), defined by the absence of estrogen receptor, progesterone receptor, and human epidermal growth factor receptor expression, remains a therapeutically challenging disease with the worst prognosis of any breast cancer subtype ${ }^{1}$.

Mammalian target of rapamycin (mTOR), a downstream protein of the PI3K/Akt pathway, is activated by growth, changes in nutrient and energy levels, and hypoxia, and has also been implicated in cancer cell proliferation and survival ${ }^{2}$. mTOR complex 1 (mTORC1) responds to intracellular energy levels and nutrient availability, and once activated, phosphorylates and activates ribosomal p70 S6 kinase (p70S6K) and hyperphosphorylates eukaryotic translation initiation factor $4 \mathrm{E}$ (eIF4E)-binding proteins 1 (4E-BP1), influencing cell growth and survival. Recent evidence has indicated that glycogen synthase kinase 3 beta (GSK3 $\beta$ ) positively regulates mTORC1 activity in MCF-7 breast cancer cells ${ }^{3}$. Moreover, GSK3 $\beta$ has been shown to play a permissive role in the amino acid-induced activation of $\mathrm{mTORC} 1^{4}$.

${ }^{1}$ Department of Medical Laboratory Science and Biotechnology, College of Health Sciences, Kaohsiung Medical University, No. 100, Shih-Chuan 1st Rd., Kaohsiung, 80702, Taiwan. ${ }^{2}$ Department of Pathology and Laboratory Medicine, Kaohsiung Veterans General Hospital, No. 386, Ta-chung 1 Rd., Kaohsiung, 81346, Taiwan. ${ }^{3}$ Division of Plastic Surgery, Department of Surgery, Taipei Veterans General Hospital, No. 201, Sec. 2, Shipai Rd., Taipei, 11221, Taiwan. ${ }^{4}$ Department of Surgery, School of Medicine, National Yang Ming University, No. 155, Sec. 2, Linong Street, Taipei, 11221, Taiwan. ${ }^{5}$ Department of Environmental and Public Healthy, School of Medicine, College of Medicine, Kaohsiung Medical University, No. 100, Shih-Chuan 1st Rd., Kaohsiung, 80702, Taiwan. Correspondence and requests for materials should be addressed to S.-M.T. (email: tsaism@kmu.edu.tw) or L.-Y.T. (email: tsliyu@kmu. edu.tw) 
(a)

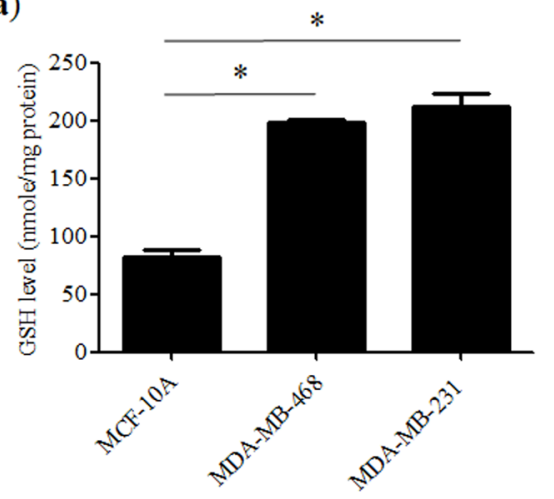

(b)

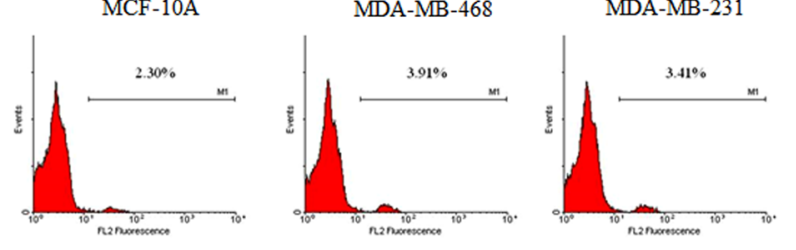

(c)

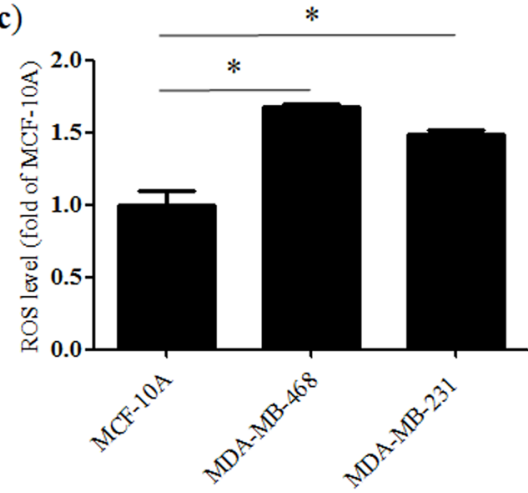

Figure 1. Redox status of various cell lines. (a) Glutathione (GSH) levels in non-tumorigenic MCF-10A cells and advanced breast cancer cell lines including MDA-MB-463 and MDA-MB-231 cells. (b) Representative flow cytometry histograms showing cellular reactive oxygen species (ROS) content and (c) a bar graph indicating fold-change relative to MCF-10A. Data represent the means \pm SD from three experiments. ${ }^{*} P<0.05$.

Reactive oxygen species (ROS) and cellular oxidative stress are associated with cancer $^{5,6}$, and compared to normal cells, antioxidant capacity is upregulated in malignant cells to adapt to higher ROS levels 5 . Previous studies have reported that nuclear factor (erythroid-derived 2)-like 2 (Nrf2) responds to increased ROS levels by enhancing the expression of genes involved in maintaining the cellular redox balance, including those encoding glutamate-cysteine ligase (GCL) and glutathione reductase (GR), which are associated with glutathione (GSH) production and regeneration ${ }^{7}$. Furthermore, deregulation of $\mathrm{Nrf} 2$, such as that leading to its increased nuclear accumulation, reduces apoptosis and promotes drug resistance ${ }^{8}$.

Since mTOR and Nrf2 signaling are known drivers of human oncogenesis ${ }^{9,10}$, agents targeting these pathways have shown promise as treatments for breast cancer ${ }^{11,12}$. However, despite the establishment of this treatment strategy and its encouraging results, a proportion of patients with TNBC harbor tumors that are resistant to mTOR inhibitors ${ }^{13}$. Thus, identifying markers of mTOR inhibitor sensitivity or the development of combination therapy is urgently needed to improve TNBC response to mTOR inhibition.

Whey protein concentrate (WPC) is prepared in a special manner to preserve native forms of the cysteine-rich proteins in whey (serum albumin, lactoferrin, and $\alpha$-lactalbumin) and functions as a GSH precursor in cells. We have shown previously that WPC supplementation increases antioxidant activity in human peripheral blood mononuclear cells and rats treated with high doses of alcohol ${ }^{14,15}$. Besides, we have demonstrated that WPC supplementation selectively depletes tumor GSH levels in 7,12-dimethylbenz[a]anthracene (DMBA)-induced mammary tumors in rats $^{16}$. In addition, prior studies have reported that WPC ingestion promotes activation of the mTOR pathway ${ }^{17}$, and it was demonstrated that this supplement exerts its antioxidant effects through an Nrf2-dependent mechanism in endothelial cells ${ }^{18}$. Therefore, in the present work, we aimed to determine whether WPC can influence the susceptibility to mTOR inhibitors using MDA-MB-231 TNBC cells, a cell line that has been reported to be resistant ${ }^{19,20}$. These results might provide insight into the critical pathways that are involved in resistance to mTOR inhibition in TNBC, and could identify biomarkers of for the responsiveness to such inhibitors.

\section{Results}

Determination of cellular redox status. Increased GSH (Fig. 1a) and ROS (Fig. 1b,c) levels were noted in advanced breast cancer cell lines, including MDA-MB-231 and MDA-MB-468, compared to those in MCF-10A non-tumorigenic breast epithelial cells. Since GSH is a key cellular antioxidant, these results indicated that cancer cells have higher tolerance to ROS. 
(a)

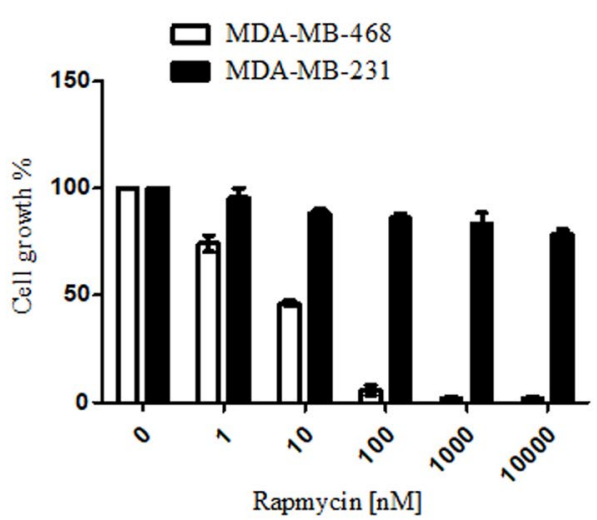

(c)

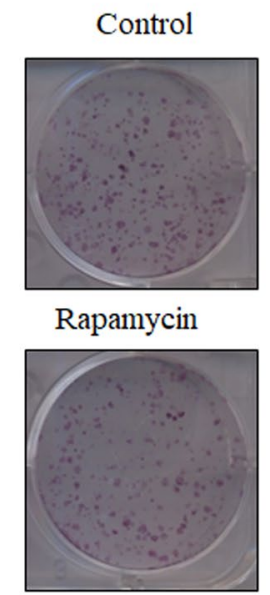

(b)

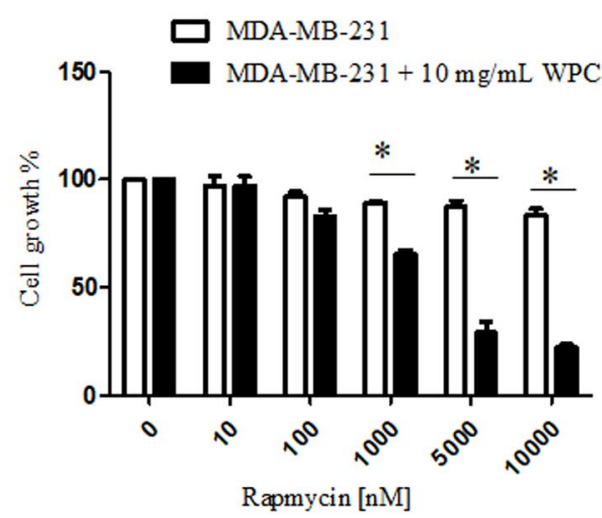

(d)

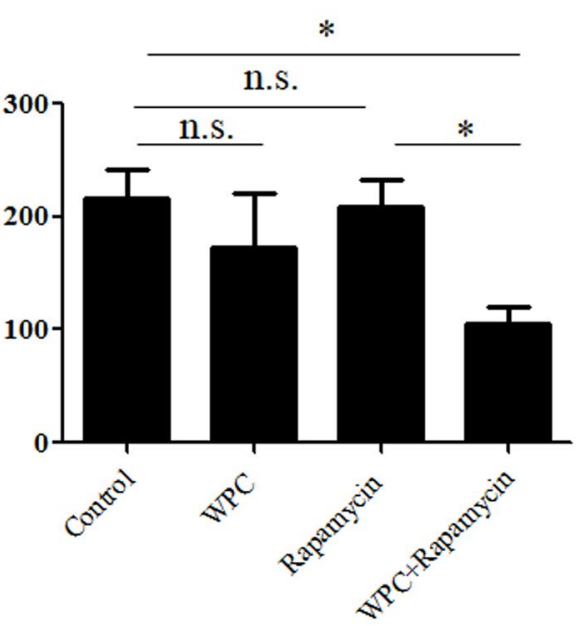

Figure 2. Whey protein concentrate (WPC) renders MDA-MB-231 cells sensitive to rapamycin and inhibits the colony formation of MDA-MB-231 cells. (a) MDA-MB-468 and MDA-MB-231 cell viability was determined by MTT assay after incubation with rapamycin at the indicated concentration for 72 hours. (b) Inhibition of MDA-MB-231 cell growth following 48 hours of exposure to $10 \mathrm{mg} / \mathrm{mL}$ WPC combined with 10, 100, 1,000, 5000 , and 10,000 nM rapamycin. (c) Representative images demonstrating the effect of WPC, rapamycin, and their combination on colony formation in MDA-MB-231 cells. (d) Bar graph indicating the number of colonies formed. Data represent the means $\pm S D$ from three experiments. $* P<0.05$; n.s.: not significant.

WPC sensitizes MDA-MB-231 cells to rapamycin. To examine the sensitivity of each cell line to rapamycin, cells were treated with this drug at different concentrations for 72 hours, and cell viability was measured by standard MTT assays. As shown in Fig. 2a, growth of MDA-MB-468 cells was significantly inhibited by rapamycin, whereas MDA-MB-231 cells were resistant to the growth inhibitory effects of this drug, with observed half maximal inhibitory concentration $\left(\mathrm{IC}_{50}\right)$ values of $8.91 \mathrm{nM}$ and $>10 \mu \mathrm{M}$, respectively. Given these different responses to rapamycin, we chose the MDA-MB-231 line for further evaluation. In addition, cellular viability of MCF-10A, MDA-MB-468, and MDA-MB-231 cells was measured with WPC at concentrations of 1,10 , and $20 \mathrm{mg} / \mathrm{mL}$. There were no significant differences $(P>0.05)$ in cell viability among the WPC-treated groups, despite increasing concentrations of WPC (Fig. S1a-c). Regarding viability of MDA-MB-231 cells, Fig. 2b shows the effect of 48 -hour exposure to WPC $(10 \mathrm{mg} / \mathrm{mL})$ combined with different concentrations of rapamycin. Compared to treatment with rapamycin alone, the growth inhibitory effect of combined treatment resulted in a significant decrease in the $\mathrm{IC}_{50}$ of rapamycin (from $>10 \mu \mathrm{M}$ to $2.54 \mu \mathrm{M}$, respectively). In vitro colony formation assays were then performed to further test the effect of WPC combined with rapamycin on cell growth. Figure $2 c$ and d demonstrate that combined treatment led to a significant decrease in the number of colonies compared to that in the control and rapamycin-only groups. However, cells treated with WPC or rapamycin alone did not significantly differ from the control in this respect.

Effect of WPC, rapamycin, and their combination on cellular redox status. As shown in Fig. 3a, WPC $(10 \mathrm{mg} / \mathrm{mL})$ treatment selectively depleted GSH levels in MDA-MB-231 cells, but increased GSH levels in non-tumorigenic MCF-10A cells. Moreover, in MDA-MB-231 cells, rapamycin $(1 \mu \mathrm{M})$ treatment did not alter GSH levels (Fig. 3b), but did significantly increase ROS concentrations (Fig. 3c,d) compared to those in the control. Furthermore, in comparison to those in the control, GSH levels were significantly lower following administration of WPC alone or in combination with rapamycin. However, WPC alone had no effect on ROS levels in 
(a)

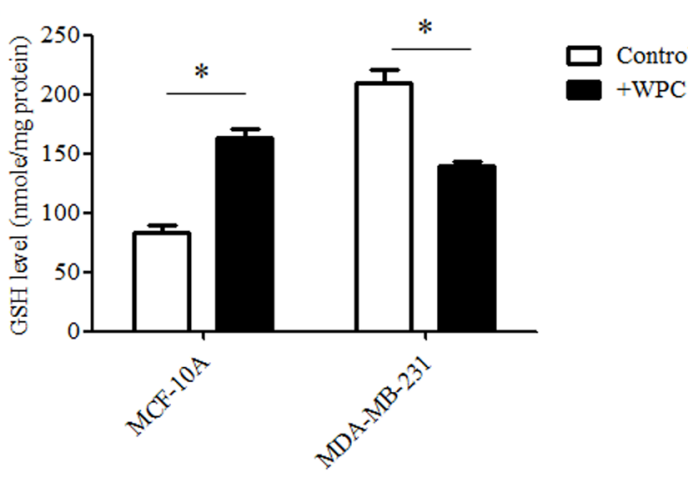

(c)
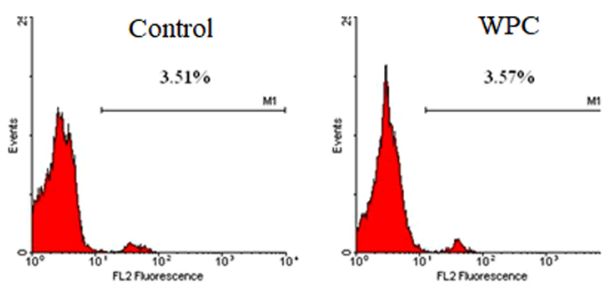

Rapamycin

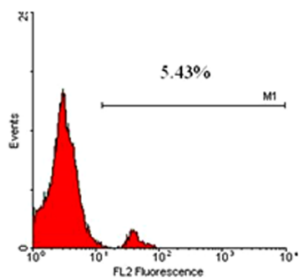

$\mathrm{WPC}+$ Rapamycin

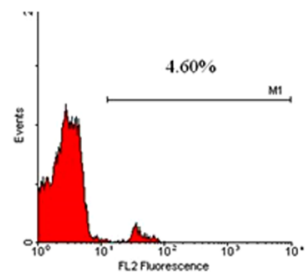

(b)

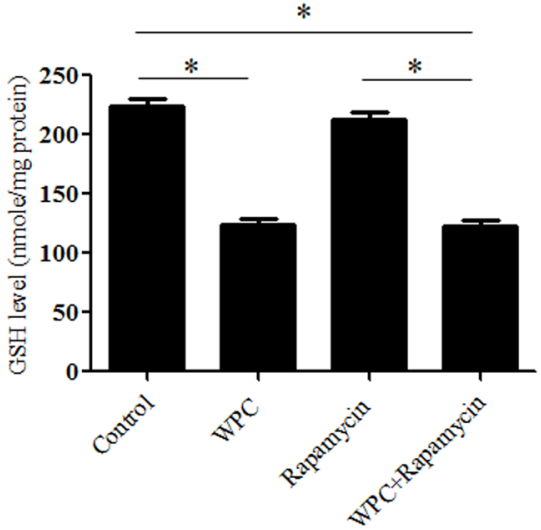

(d)

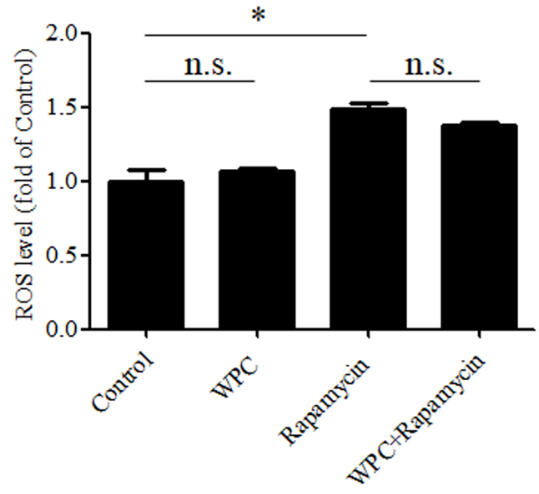

Figure 3. Effect of whey protein concentrate (WPC), rapamycin, and their combination on the redox status of breast cancer cells. (a) Glutathione (GSH) levels in non-tumorigenic MCF-10A cells and MDA-MB-231 cells after incubation with $10 \mathrm{mg} / \mathrm{mL}$ WPC for 48 hours. Effect of 48 -hour treatment with $10 \mathrm{mg} / \mathrm{mL} \mathrm{WPC}$, $1 \mu \mathrm{M}$ rapamycin, and their combination on (b) GSH levels and (c) reactive oxygen species (ROS) content in MDA-MB-231 cells, displayed as representative flow cytometry histograms and (d) a bar graph indicating foldchange relative to control levels. Data represent the means $\pm S D$ from three experiments. ${ }^{*} P<0.05$; n.s.: not significant.

MDA-MB-231 cells and did not affect the rapamycin-mediated elevation in ROS concentrations when administered as part of the combination treatment.

Effect of WPC, rapamycin, and their combination on the expression of Nrf2, GCL, and GR in MDA-MB-231 cells. Since the intracellular GSH redox state is regulated by the Nrf2-GCL-GR-GSH signaling pathway, whole cell lysates were subjected to immunoblotting for Nrf2, the GCL catalytic subunit (GCLC), and GR to clarify the effects of WPC. As shown in Fig. 4, there were no significant differences $(\mathrm{P}>0.05)$ in whole-cell Nrf2 protein expression between groups with WPC treatment alone or combined treatment, when compared to that in the control group. However, treatment with rapamycin alone increased whole-cell Nrf2 protein expression. Compared to the that in the control group, WPC alone and combined treatment resulted in reduced expression of GCLC and GR proteins. However, treatment with rapamycin alone had no effect on the expression of these proteins.

Rapamycin-induced nuclear accumulation of Nrf2 in MDA-MB-231 cells is reduced by WPC treatment. Since there were no significant differences in whole-cell Nrf2 protein expression among groups, we next evaluated the cellular translocation of Nrf2. As indicated in Fig. 5a and b, rapamycin treatment induced Nrf2 nuclear accumulation; however, WPC administration decreased the presence of Nrf2 in the nucleus, and inhibited the effect of rapamycin on Nrf2 translocation.

WPC activates the GSK3/mTOR pathway. We next assessed the combined effects of WPC and rapamycin on mTORC1 in MDA-MB-231 cells, since this complex is the direct target of rapamycin and is activated by nutritional factors. The level of phosphorylated (p)-mTOR (Ser2448) is a marker of mTORC1 activation. As shown in Fig. 6, the p-mTOR/mTOR ratio was approximately 2.2-fold higher in the WPC-only group compared to that in the control group. In contrast, compared to that in the control, the $\mathrm{p}-\mathrm{mTOR} / \mathrm{mTOR}$ ratio was found to be reduced with combined treatment. In addition, the p-p70S6K/p70S6K and the p-4E-BP1/4E-BP1 ratios were 


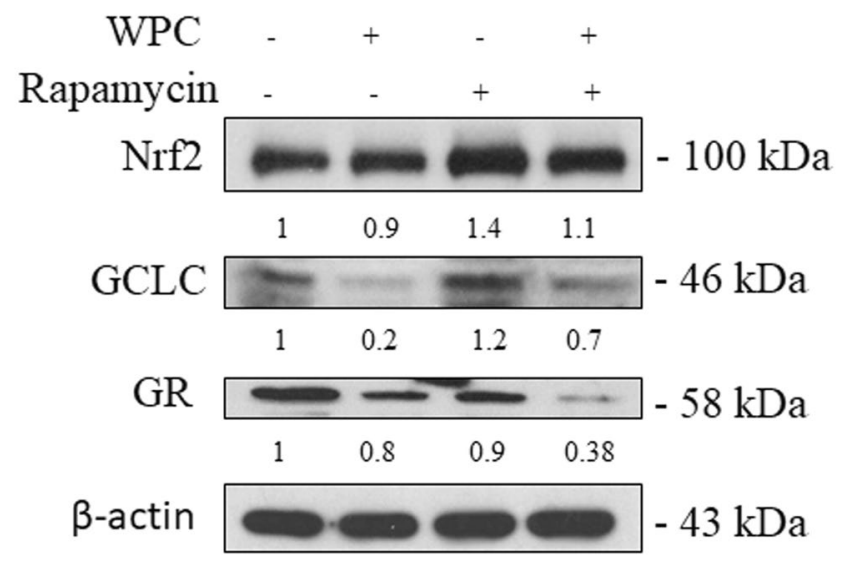

\section{Whole cell lysates}

Figure 4. Effect of whey protein concentrate (WPC), rapamycin, and their combination on the expression of Nrf2, GCLC, and glutathione reductase (GR) proteins in MDA-MB-231 cells. Representative western blots showing the effect of 48 hours of exposure to $10 \mathrm{mg} / \mathrm{mL}$ WPC, $1 \mu \mathrm{M}$ rapamycin, and their combination on whole cell Nrf2, GCLC, and GR protein levels. $\beta$-actin was used as a loading control. Data represent the means \pm SD from three experiments.

(a)

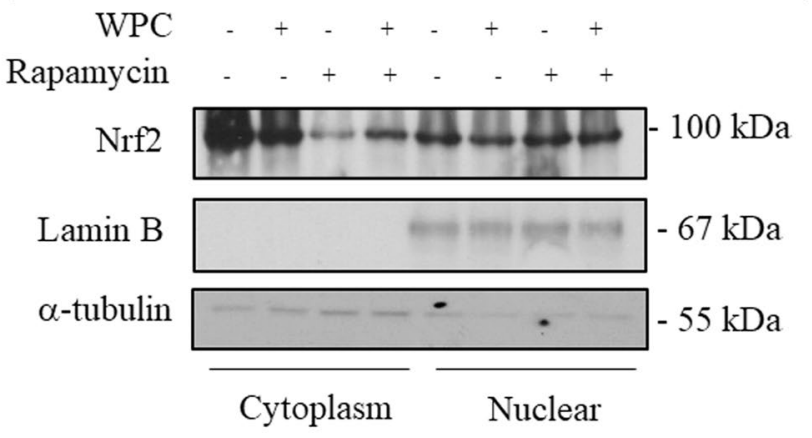

(b)

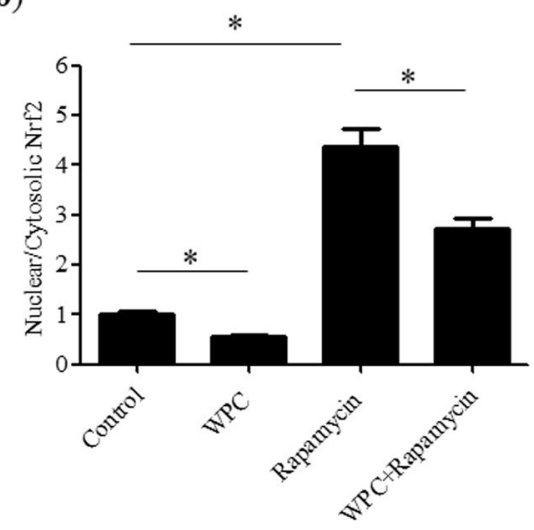

Figure 5. Effect of when protein concentrate (WPC), rapamycin, and their combination on Nrf2 translocation in MDA-MB-231 cells. (a) Representative western blots showing the effect of 48 hours of exposure to $10 \mathrm{mg} /$ $\mathrm{mL}$ WPC, $1 \mu \mathrm{M}$ rapamycin, and their combination on cytosolic and nuclear Nrf2 protein levels. $\alpha$-tubulin and lamin B were used as loading controls for cytosolic and nuclear fractions, respectively. (b) Bar graphs showing relative nuclear/cytosolic Nrf2 ratios. Data represent the means \pm SD from three experiments. $* P<0.05$.

1.4-fold and 1.5-fold higher, respectively, in the WPC-only group than the control group, whereas this effect was negated by the administration of rapamycin together with WPC. Strikingly, relative to that in the control, WPC upregulated p-GSK3 $\beta /$ GSK3 $\beta$, both alone (1.32-fold) and with combined treatment (2.2-fold).

WPC reduces Nrf2 nuclear accumulation and induces mTORC1 activation via GSK3 $\beta$ activity. To determine the mechanism underlying the WPC-mediated reduction in Nrf2 nuclear accumulation, we downregulated GSK3 $\beta$ protein expression using siRNA specific to the corresponding mRNA. Cells were transfected with non-specific (scrambled) siRNA or GSK3 $\beta$ siRNA, and were then treated with WPC. Protein expression was then detected by immunoblotting (Fig. 7a). GSK3 $\beta$ was inhibited, as expected, and this was found to result in increased nuclear Nrf2, compared to cytosolic levels (Fig. 7b,c). Moreover, cells treated with GSK3 $\beta$ siRNA exhibited a reduced $\mathrm{p}-\mathrm{mTOR} / \mathrm{mTOR}$ ratio in response to WPC treatment (Fig. 7d,e).

\section{Discussion}

Although many studies have investigated molecular markers such as VEGF ${ }^{21}, \mathrm{EGFR}^{22}$, and $\mathrm{mTOR}^{23}$ as drug targets for TNBC, a greater understanding of the molecular basis of this disease is required for the development of effective treatments. In the present study, we demonstrated that WPC treatment renders MDA-MB-231 cells susceptible to rapamycin by (i) altering the cellular redox state and (ii) activating GSK3 $3 / \mathrm{mTORC} 1$ signaling. 


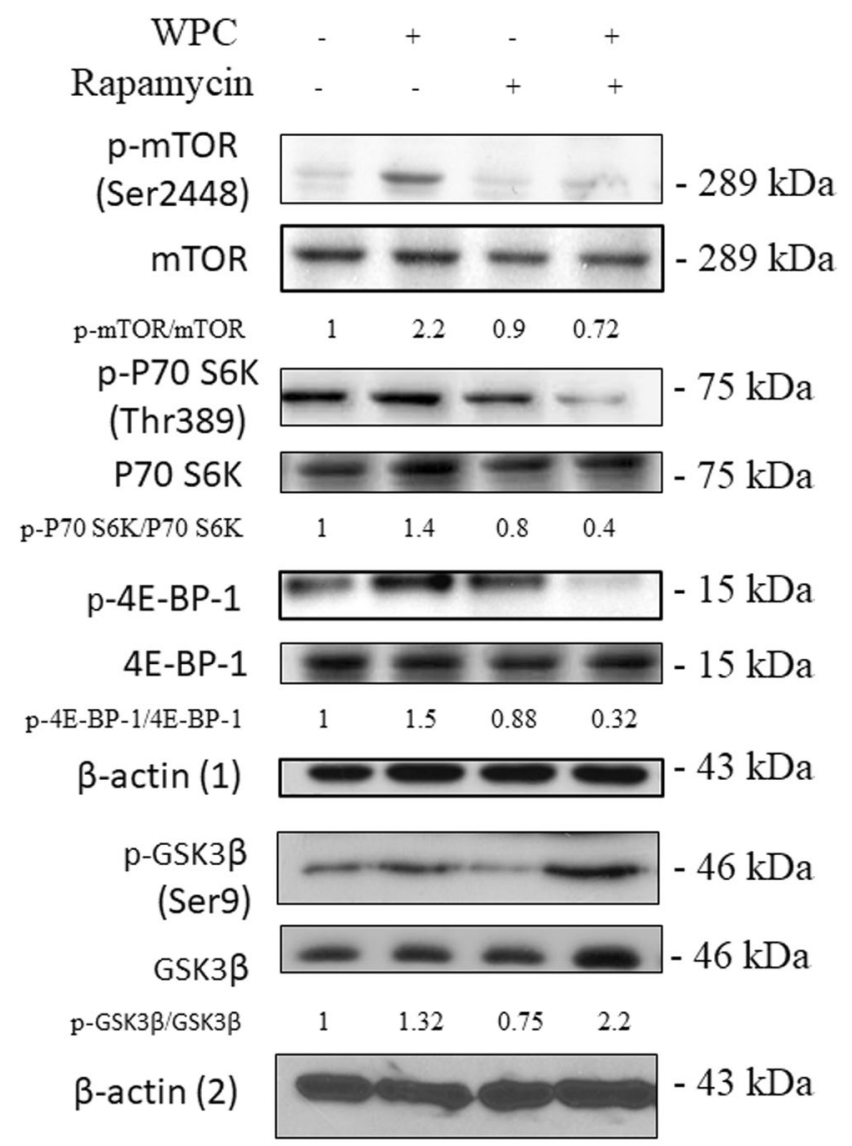

Figure 6. Effect of whey protein concentrate (WPC), rapamycin, and their combination on the mTOR/GSK3 $\beta$ signaling pathway in MDA-MB-231 cells. Representative western blots showing levels of p-mTOR (Ser 2448), mTOR, p-p70S6K (Thr 389), p70S6K, p-4E-BP-1, 4E-BP-1, p-GSK3 $\beta$, and GSK3 $\beta$ in cells treated with $10 \mathrm{mg} /$ $\mathrm{mL}$ WPC, $1 \mu \mathrm{M}$ rapamycin, or both for 48 hours. $\beta$-actin (1) was used as a loading control of p-mTOR (Ser 2448), mTOR, p-p70S6K (Thr 389), p70S6K, p-4E-BP-1, and 4E-BP-1. $\beta$-actin (2) was used as a loading control of $\mathrm{p}-\mathrm{GSK} 3 \beta$, and GSK3 $\beta$ Data represent the means \pm SD from three experiments.

Basal-like TNBC cells were reported to be highly sensitive to the mTOR inhibitor everolimus, whereas a subgroup of TNBC cells, comprising lines characterized as stem-like cancer cells, are less sensitive to this drug ${ }^{24}$. Given that the cellular redox state affects the regulation of signal transduction pathways and the development of drug resistance, this could be used to dictate therapeutic strategies for $\mathrm{TNBC}^{25}$. Many studies have shown that redox mechanisms regulate $\mathrm{mTORC} 1$ activity $^{26}$. For example, Sarbassov et al. demonstrated that cysteine oxidants such as phenylarsine oxide and diamide activate mTORC1 in vivo and in vitro ${ }^{27}$.

In the current work, we first observed that GSH and ROS levels were elevated in MDA-MB-231 cells compared to those in non-tumorigenic MCF-10A cells. Our previous results indicated an imbalance in GSH redox status in breast cancer patients. The high GSH levels observed in breast cancer tissues might be associated with enhanced cell proliferation and resistance to oxidative stress, representing a selective growth advantage for tumor cells over their normal counterparts ${ }^{28}$. In addition, Panis et al. reported that compared to adjacent normal breast tissue, tumors are sites of elevated oxidative and nitrosative stress, with concomitant augmented antioxidant capacity ${ }^{29}$.

WPC constitutes a rich source of bioavailable cysteine that can be used for GSH synthesis, and contains all nine essential amino acids. It is used to promote muscle protein synthesis and as a nutritional supplement for chemotherapy patients ${ }^{30}$. The PTEN-positive cell line MDA-MB-231 has been reported to be resistant to mTOR inhibition; however, we observed here that WPC treatment rendered these cells sensitive to rapamycin. Furthermore, WPC selectively altered GSH levels, affecting those in MDA-MB-231 cells, but not those in non-tumorigenic MCF-10A cells. This might be explained by the fact that GSH synthesis is regulated by negative feedback. Since GSH levels are much higher in tumor cells than in normal cells, it is easier to reach the threshold at which GSH synthesis is inhibited by negative feedback in the former than in the latter ${ }^{31,32}$. In addition, we found that treatment with $1 \mu \mathrm{M}$ rapamycin, with or without WPC, induces ROS production in MDA-MB-231 cells. The relationship between rapamycin and ROS remains disputed, with some studies suggesting that rapamycin reduces cellular ROS levels ${ }^{33}$ and others claiming the opposite ${ }^{34}$. Such discrepancies might be explained by the important effect of rapamycin concentration ${ }^{35,36}$. In addition, there were no obvious changes in the viability of cells with WPC treatment. It is notable that in the present work, WPC selectively affected GSH levels but did not change cellular ROS content and viability, implying that a synergistic antitumor effect might be achieved by decreasing GSH levels in tandem with WPC treatment ${ }^{37}$. 
(a)

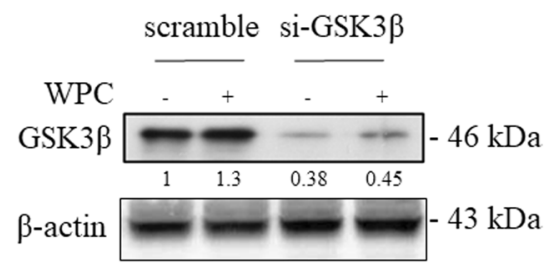

(b)

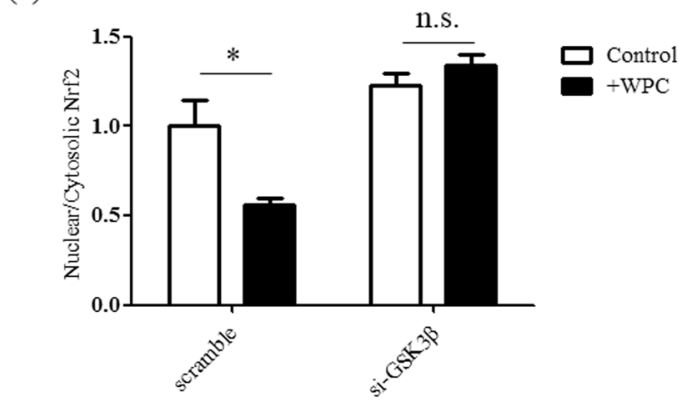

(d)

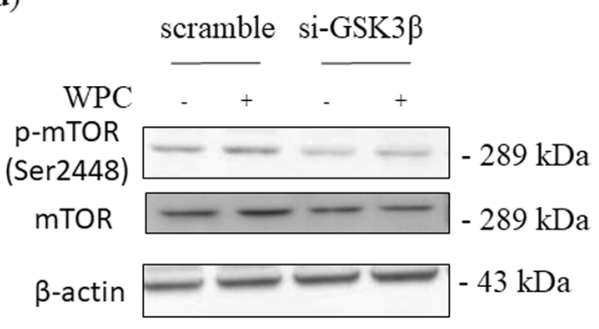

(e)

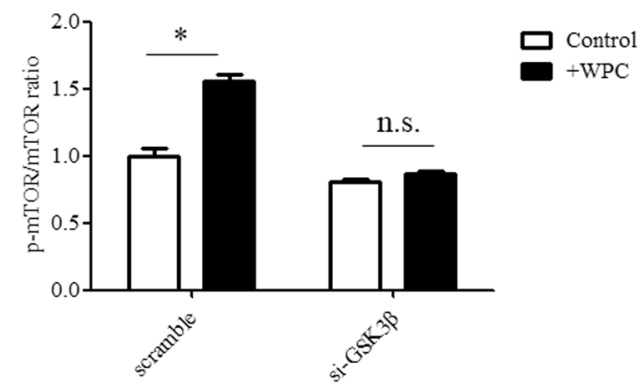

Figure 7. Whey protein concentrate (WPC)-induced reduction of Nrf2 nuclear accumulation and mTORC1 activation are disrupted by GSK3 $\beta$ siRNA. MDA-MB-231 cells were transfected with non-specific siRNA or GSK3 $\beta$-targeting siRNA and treated for 48 hours with $10 \mathrm{mg} / \mathrm{mL}$ WPC. (a) Representative western blots showing GSK3 $\beta$ protein levels. $\beta$-actin was used as a loading control. (b) Representative western blots showing cytosolic and nuclear levels of Nrf2. Lamin B and $\alpha$-tubulin were used as loading controls for nuclear and cytosolic fractions, respectively. (c) Bar graphs showing relative nuclear/cytosolic Nrf2 ratios. (d) Representative western blots demonstrating levels of p-mTOR (Ser2448) and mTOR. $\beta$-actin was used as a loading control. (e) Bar graphs displaying relative $\mathrm{p}-\mathrm{mTOR} / \mathrm{mTOR}$ ratios. Data represent the means $\pm \mathrm{SD}$ from three experiments. $* P<0.05 ;$ n.s.: not significant.

Nrf2 regulates the inducible antioxidant program in response to cellular stress ${ }^{38}$. Here, we revealed that rapamycin treatment induces the nuclear accumulation of $\mathrm{Nrf} 2$, consistent with the observations of other researchers. It has also been reported that TORC1 inhibition by rapamycin increases transcription of the Nrf2 gene ${ }^{39,40}$. Notably, combining rapamycin with WPC negated the promotion of Nrf2 nuclear accumulation by the rapamycin treatment in the present study. Moreover, we observed reduced expression of GCLC and GR proteins when cells were treated with WPC. Consistent with the observations of our previous in vivo study, we found that WPC supplementation reduced the nuclear accumulation of Nrf2 in tumor tissue ${ }^{16}$. Kerasioti et al. indicated that whey protein exerts its antioxidant activity through an Nrf2-dependent mechanism in endothelial cells; however, it was cell type dependent ${ }^{18}$.

It has been suggested that $\mathrm{p}-\mathrm{p} 70 \mathrm{~S} 6 \mathrm{~K}^{\mathrm{low}} / \mathrm{p}-4 \mathrm{E}-\mathrm{BP} 1^{\text {high }}$ cancer cells might be intrinsically resistant to mTORC1 inhibitors ${ }^{41}$. Of note, we found here that WPC treatment reversed this phenotype by enhancing phosphorylation of mTOR (Ser2448), p70S6K (Thr389), and 4E-BP1. Furthermore, we revealed that WPC upregulated GSK3 $\beta$ expression, consistent with a study by Stretton et al., in which GSK3 $\beta$ activity was shown to be required for the support of mTORC1 signaling in response to nutrient availability ${ }^{4}$. It has also been recognized that amino acid treatment promotes mTORC1 activation ${ }^{42,43}$. Our investigation suggests a combined therapy that might be beneficial for the subgroup of TNBC patients with tumors resistant to mTOR inhibitors.

Finally, in the present study, we inhibited GSK3 $\beta$ activity using siRNA, the results of which suggested that GSK $3 \beta$ is required for the reduction in Nrf2 nuclear accumulation mediated by WPC treatment. This is consistent with a report that activation and direct inhibition of GSK3 $\beta$ leads to nuclear exclusion and accumulation of Nrf2, respectively ${ }^{44}$. In addition, GSK3 $\beta$ was found to be associated with WPC-induced mTORC1 activation. Taken together, our results indicate that WPC leads to the depletion of GSH by reducing Nrf2 nuclear accumulation and activating GSK3 $3 / \mathrm{mTOR}$ signaling, rendering MDA-MB-231 cells susceptible to rapamycin. 


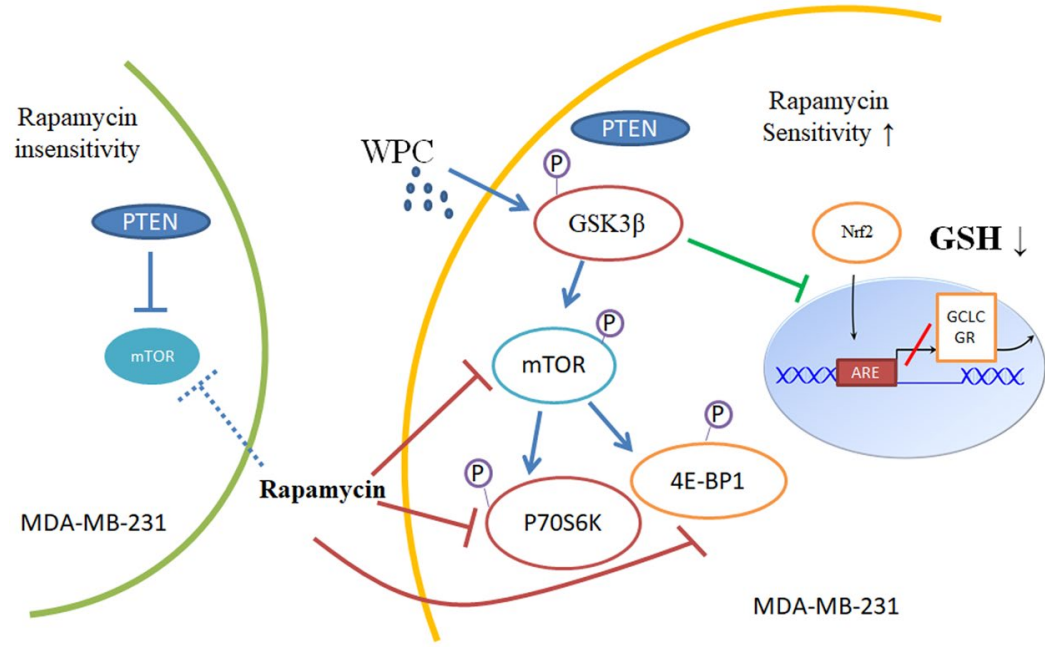

Figure 8. A working model of the mechanism through which whey protein concentrate (WPC) renders MDA-MB-231 cells sensitive to rapamycin. MDA-MB-231 cells express wild-type PTEN and are insensitive to rapamycin. WPC treatment activates mTOR/GSK3 $\beta$ signaling, involving increased p-p70S6K and p-4E-BP1 levels. In addition, activation of GSK $3 \beta$ inhibits translocation of Nrf2 from the cytoplasm to the nucleus, altering the cellular redox state by downregulating the glutamate-cysteine ligase catalytic subunit (GCLC) and glutathione reductase (GR), and finally reducing glutathione (GSH) levels. Taking together, WPC treatment thus induces rapamycin sensitivity in MDA-MB-231 cells.

In conclusion, we explored the result of combining antioxidant and mTOR inhibitory effects, and clarified the underlying mechanism for the first time (Fig. 8). Our findings might serve as a reference for the development of such combination therapies for the treatment of breast cancer, and particularly TNBC.

\section{Materials and Methods}

Reagents and antibodies. The WPC (Immunocal, Immunotec Research Ltd., Quebec, Canada) contained about $90 \%$ whey protein isolate, $<1.5 \%$ lactose, $<0.5 \%$ fat, and $<5.0 \%$ moisture, with a solubility index of $99 \%$ at $\mathrm{pH}$ 4.6. WPC was freshly prepared before each experiment. The rapamycin and dimethylsulfoxide (DMSO) were purchased from Sigma-Aldrich (St. Louis, MO, USA). DMSO used as a solvent for rapamycin and control cells, which was not beyond $0.1 \%$. The antibodies used in this experiment included: GCLC (abcam, ab53179), GR (abcam, ab16801), Nrf2 (Cell Signaling Technology (CST), \#12721), mTOR (CST, \#2972), p-mTOR (Ser 2448) (CST, \#2971), p70S6K (CST, \#9202), p-p70S6K (Thr389) (CST, \#9205), 4E-BP1 (CST, \#9644), p-4E-BP1 (Thr 37/46) (CST, \#2855), GSK3 $\beta$ (CST, \#9332), p-GSK3 $\beta$ (Ser9) (CST, \#9336), $\beta$-actin (Sigma, A5441), $\alpha$-tubulin (Santa Cruz Biotechnology, sc-8035), and Lamin B (sc-6216).

Cell culture. The TNBC cell lines, MDA-MB-231 and MDA-MB-468 were obtained from American Type Culture Collection (Manassas, VA, USA). The nonmalignant MCF-10A breast epithelial cells were a kind gift from Taipei Medical University Hospital. MDA-MB-231 and MDA-MB-468 cells were cultured in DMEM supplemented with $10 \%$ fetal bovine serum (FBS), and MCF-10A cells were maintained in DMEM/F-12 medium supplemented with $5 \%$ horse serum, $0.5 \mu \mathrm{g} / \mathrm{mL}$ hydrocortisone, $100 \mathrm{ng} / \mathrm{ml}$ cholera toxin, $10 \mu \mathrm{g} / \mathrm{mL}$ insulin, and $20 \mathrm{ng} / \mathrm{ml}$ recombinant human EGF. All cells were cultured in a humidified incubator containing $5 \% \mathrm{CO}_{2}$ at $37^{\circ} \mathrm{C}$.

Preparation of WPC. Preparation of WPC was the same as in our previous study ${ }^{15}$. We followed the instructions of Immunocal to design the concentrations of WPC and WPC was freshly prepared before each experiment. Briefly, $50 \mathrm{mg}$ of WPC was dissolved in a fresh $5 \mathrm{~mL}$ serum-free medium and centrifuged at $12,000 \times g$ for $10 \mathrm{~min}$ utes at room temperature. The supernatant was filtered with a $0.2 \mu \mathrm{m}$ filter. Therefore, concentrations of $10 \mathrm{mg} /$ $\mathrm{mL}$ of WPC were used in this experiment.

Determination of GSH. The $100 \mu \mathrm{L}$ cell suspension, as indicated above, in an amber microcentrifuge tube was mixed with $0.1 \%$ MPA in a 1:2 volumetric ratio, and then it was centrifuged at $12,000 \times g$ for 10 minutes after being adequately mixed by a vortex mixer. The supernatant was assayed on a capillary electrophoresis analyzer (P/ACE MDQ, Beckman Coulter) after being filtered with a $0.2 \mu \mathrm{m}$ syringe set. The analysis was performed at a constant temperature $\left(28^{\circ} \mathrm{C}\right)$ with $300 \mathrm{mM}$ borate running buffer $(\mathrm{pH} 7.8)$ and a UV absorbance detector set to $200 \mathrm{~nm}$.

Flow cytometric analysis of ROS levels. The intracellular ROS levels were assessed by flow cytometry using DCFH-DA. To assess the ROS levels, the cells were resuspended in PBS at 1,000,000 cells per mL and incubated in the presence of DCFH-DA $(10 \mu \mathrm{M})$ in the dark at $37^{\circ} \mathrm{C}$ for 30 minutes. Then, the cells were washed and resuspended in PBS. Finally, submitted the sample into flow cytometric analysis using a FACScan flow cytometer (Coulter EPICS XL Flow Cytometer, Beckman Coulter). Analyses were performed on 10,000 cells per sample and 
fluorescence intensities were measured on a logarithmic scale of four decades of log of fluorescence. Each experiment was repeated at least three times.

Cell viability assays. Cell viability was assessed by 3-(4,5-Dimethylthiazol-2-yl)-2,5-diphenyltetrazolium bromide (MTT) dye conversion at $570 \mathrm{~nm}$ following the manufacturer's instructions. Briefly, cells were seeded 5,000 per well in a 96-well flat bottom plate and grown for 24 hours. Cells were then treated for 48 hours or 72 hours with indicated concentrations of rapamycin and WPC in the presence of 10\% FBS. $20 \mu \mathrm{lof}$ MTT (5 mg/ $\mathrm{mL}$ in PBS) was then added to each well and incubated 2 hours at $37^{\circ} \mathrm{C}$. The formazan in viable cells were dissolved with $100 \mu \mathrm{l}$ of dimethyl sulfoxide and determined by reading optical densities in a microplate reader (Dynatech Laboratories, Chantilly, VA, USA).

Colonies formation assay. MDA-MB-231 cells were seeded in 6-well plate at a density of 1,500 cells per well duplicate. These cells were cultured with respective treatment including WPC $(2 \mathrm{mg} / \mathrm{mL})$, rapamycin $(200 \mathrm{nM})$, and their combination. The medium was replaced every $2-3$ days until day 14 . Colonies were stained with $0.2 \%(\mathrm{w} / \mathrm{v})$ crystal violet in buffered formalin for 10 minutes. The number of colonies was counted.

Preparation of cytosolic and nuclear extracts. Cytosolic and nuclear extracts were isolated according to Schreiber et al. ${ }^{45}$ with some modify cations. After rinsing with cold PBS, cells were resuspended in cold lysis buffer A (20 mM HEPES pH 8.0, 1 mM EDTA, $1.5 \mathrm{mM} \mathrm{MgCl}_{2}, 10 \mathrm{mM} \mathrm{KCl,} 1 \mathrm{mM}$ DTT, $1 \mathrm{mM}$ sodium orthovanadate, $1 \mathrm{mM}$ NaF, $1 \mathrm{mM}$ PMSF, $0.5 \mathrm{mg} / \mathrm{mL}$ benzamidine, $0.1 \mathrm{mg} / \mathrm{ml}$ leupeptin, and $1.2 \mathrm{mg} / \mathrm{mL}$ aprotinin). They were incubated on ice for $15 \mathrm{~min}$, after which $7.5 \mu \mathrm{L}$ of $10 \% \mathrm{NP}-40$ detergent was added. Afterwards the cells were intensively stirred in a vortex mixer for $10 \mathrm{~s}$. The homogenate was centrifuged at $15,000 \times g$ for 50 seconds, and the supernatant was used as the cytosolic extract. The nuclear pellet was resuspended in cold extraction buffer B (20 mM HEPES pH 8.0), $1 \mathrm{mM}$ EDTA, $1.5 \mathrm{mM} \mathrm{MgCl}_{2}, 10 \mathrm{mM} \mathrm{KCl}, 1 \mathrm{mM}$ DTT, $1 \mathrm{mM}$ sodium orthovanadate, $1 \mathrm{mM}$ NaF, $1 \mathrm{mM}$ PMSF, $0.5 \mathrm{mg} / \mathrm{mL}$ benzamidine, $0.1 \mathrm{mg} / \mathrm{mL}$ leupeptin, $1.2 \mathrm{mg} / \mathrm{mL}$ aprotinin, and $20 \%$ glycerol). All protein fractions were stored at $-70^{\circ} \mathrm{C}$ until used.

Western blotting. Cells were seeded, cultured, and treated with WPC $(10 \mathrm{mg} / \mathrm{mL})$, rapamycin $(1 \mu \mathrm{M})$, and their combination for 48 hours. The proteins were separated by SDS-PAGE and transferred to polyvinylidene difluoride (PVDF) membrane. The membranes were blocked with 5\% milk for 1 hour and incubated with targeted primary antibodies overnight at $4{ }^{\circ} \mathrm{C}$, and then incubated with HRP-conjugated secondary antibodies at room temperature for 1 hour. Finally, the signals were enhanced by ECL (Millipore, Temecula, CA, USA) and exposed to X-ray film for autoradiogram. The intensity of the bands was quantified by densitometry and the results were evaluated relative to the expression of the constitutively expressed protein $\beta$-actin.

Small interfering RNA (siRNA). GSK-3 $\beta$ was silenced using siRNA (Santa Cruz Biotechnology, sc-35527) and nonspecific scrambled siRNA (sc-37007) used as the negative control. Cells at $80 \%$ confluency were transfected with $2 \mu \mathrm{g}$ of siRNA/well in 6-well plates using lipofectamine 2000 (Invitrogen). After they had been transfected, the cells were incubated for 18 hours in DMEM supplemented with $10 \% \mathrm{FBS}$ at $37^{\circ} \mathrm{C}$, then treated with WPC or rapamycin for additional 48 hours.

Statistical analysis. Data were expressed as mean \pm standard deviation (SD) from three experiments. P-values were determined by Student's $t$-test or one-way analysis of variance (ANOVA), followed by the Bonferroni post-test. Statistics were calculated using GraphPad Prism 5.0 software. Values of $P<0.05$ was considered statistically significant.

\section{References}

1. Foulkes, W. D., Smith, I. E. \& Reis-Filho, J. S. Triple-negative breast cancer. N Engl J Med 363, 1938-1948, https://doi.org/10.1056/ NEJMra1001389 (2010)

2. Sengupta, S., Peterson, T. R. \& Sabatini, D. M. Regulation of the mTOR complex 1 pathway by nutrients, growth factors, and stress. Mol Cell 40, 310-322, https://doi.org/10.1016/j.molcel.2010.09.026 (2010).

3. Azoulay-Alfaguter, I., Elya, R., Avrahami, L., Katz, A. \& Eldar-Finkelman, H. Combined regulation of mTORC1 and lysosomal acidification by GSK-3 suppresses autophagy and contributes to cancer cell growth. Oncogene 34, 4613-4623, https://doi. org/10.1038/onc.2014.390 (2015).

4. Stretton, C. et al. GSK3-mediated raptor phosphorylation supports amino-acid-dependent mTORC1-directed signalling. Biochem J 470, 207-221, https://doi.org/10.1042/bj20150404 (2015).

5. Liou, G. Y. \& Storz, P. Reactive oxygen species in cancer. Free Radic Res 44, 479-496, https://doi.org/10.3109/10715761003667554 (2010).

6. Schieber, M. \& Chandel, N. S. ROS function in redox signaling and oxidative stress. Current biology: CB 24, R453-462, https://doi. org/10.1016/j.cub.2014.03.034 (2014).

7. Zhang, Q., Pi, J., Woods, C. G. \& Andersen, M. E. A systems biology perspective on Nrf2-mediated antioxidant response. Toxicol Appl Pharmacol 244, 84-97, https://doi.org/10.1016/j.taap.2009.08.018 (2010).

8. Niture, S. K., Khatri, R. \& Jaiswal, A. K. Regulation of Nrf2-an update. Free Radic Biol Med 66, 36-44, https://doi.org/10.1016/j. freeradbiomed.2013.02.008 (2014).

9. Owonikoko, T. K. \& Khuri, F. R. Targeting the PI3K/AKT/mTOR pathway: biomarkers of success and tribulation. American Society of Clinical Oncology educational book. American Society of Clinical Oncology. Meeting, https://doi.org/10.1200/EdBook_AM.2013.33. e395 (2013).

10. Jaramillo, M. C. \& Zhang, D. D. The emerging role of the Nrf2-Keap1 signaling pathway in cancer. Genes Dev 27, 2179-2191, https:// doi.org/10.1101/gad.225680.113 (2013).

11. Paplomata, E. \& O’Regan, R. The PI3K/AKT/mTOR pathway in breast cancer: targets, trials and biomarkers. Therapeutic advances in medical oncology 6, 154-166, https://doi.org/10.1177/1758834014530023 (2014).

12. Davis, N. M. et al. Deregulation of the EGFR/PI3K/PTEN/Akt/mTORC1 pathway in breast cancer: possibilities for therapeutic intervention. Oncotarget 5, 4603-4650, https://doi.org/10.18632/oncotarget.2209 (2014). 
13. Zhang, W. L. et al. Molecular mechanisms of resistance to phosphatidyl inositol 3-kinase inhibitors in triple-negative breast cancer cells. Zhonghua zhong liu za zhi [Chinese journal of oncology] 38, 578-588, https://doi.org/10.3760/cma.j.issn.0253-3766.2016.08.004 (2016).

14. Tseng, Y. M. et al. Effects of whey protein concentrate (WPC) on the distributions of lymphocyte subpopulations in rats with excessive alcohol intake. J Agric Food Chem 58, 12729-12734, https://doi.org/10.1021/jf103518u (2010).

15. Tseng, Y. M. et al. Effects of alcohol-induced human peripheral blood mononuclear cell (PBMC) pretreated whey protein concentrate (WPC) on oxidative damage. J Agric Food Chem 56, 8141-8147, https://doi.org/10.1021/jf801034k (2008).

16. Cheng, S. H., Tseng, Y. M., Wu, S. H., Tsai, S. M. \& Tsai, L. Y. Selective effects of whey protein concentrate on glutathione levels and apoptosis in rats with mammary tumors. Food Chem Toxicol 107, 440-448, https://doi.org/10.1016/j.fct.2017.07.024 (2017).

17. Mitchell, C. J. et al. Consumption of Milk Protein or Whey Protein Results in a Similar Increase in Muscle Protein Synthesis in Middle Aged Men. Nutrients 7, 8685-8699, https://doi.org/10.3390/nu7105420 (2015).

18. Kerasioti, E., Stagos, D., Tzimi, A. \& Kouretas, D. Increase in antioxidant activity by sheep/goat whey protein through nuclear factorlike 2 (Nrf2) is cell type dependent. Food Chem Toxicol 97, 47-56, https://doi.org/10.1016/j.fct.2016.08.022 (2016).

19. Lehmann, B. D. et al. Identification of human triple-negative breast cancer subtypes and preclinical models for selection of targeted therapies. J Clin Invest 121, 2750-2767, https://doi.org/10.1172/jci45014 (2011).

20. Noh, W. C. et al. Determinants of rapamycin sensitivity in breast cancer cells. Clin Cancer Res 10, 1013-1023 (2004).

21. Burstein, H. J. et al. Phase II study of sunitinib malate, an oral multitargeted tyrosine kinase inhibitor, in patients with metastatic breast cancer previously treated with an anthracycline and a taxane. J Clin Oncol 26, 1810-1816, https://doi.org/10.1200/ jco.2007.14.5375 (2008).

22. Manupati, K. et al. Inhibiting Epidermal Growth Factor Receptor Signalling Potentiates Mesenchymal-Epithelial Transition of Breast Cancer Stem Cells and their Responsiveness to Anticancer Drugs. Febs $j$. https://doi.org/10.1111/febs.14084 (2017)

23. Dey, N., De, P. \& Leyland-Jones, B. PI3K-AKT-mTOR inhibitors in breast cancers: From tumor cell signaling to clinical trials. Pharmacology \& therapeutics, https://doi.org/10.1016/j.pharmthera.2017.02.037 (2017).

24. Miller, T. W., Balko, J. M. \& Arteaga, C. L. Phosphatidylinositol 3-kinase and antiestrogen resistance in breast cancer. J Clin Oncol 29, 4452-4461, https://doi.org/10.1200/jco.2010.34.4879 (2011).

25. Kim, D., Koo, J. S. \& Lee, S. Overexpression of reactive oxygen species scavenger enzymes is associated with a good prognosis in triple-negative breast cancer. Oncology 88, 9-17, https://doi.org/10.1159/000358365 (2015).

26. Yoshida, S. et al. Redox regulates mammalian target of rapamycin complex 1 (mTORC1) activity by modulating the TSC1/TSC2Rheb GTPase pathway. J Biol Chem 286, 32651-32660, https://doi.org/10.1074/jbc.M111.238014 (2011).

27. Sarbassov, D. D. \& Sabatini, D. M. Redox regulation of the nutrient-sensitive raptor-mTOR pathway and complex. J Biol Chem 280, 39505-39509, https://doi.org/10.1074/jbc.M506096200 (2005).

28. Yeh, C. C. et al. A study of glutathione status in the blood and tissues of patients with breast cancer. Cell Biochem Funct 24, 555-559, https://doi.org/10.1002/cbf.1275 (2006).

29. Panis, C. et al. Can Breast Tumors Affect the Oxidative Status of the Surrounding Environment? A Comparative Analysis among Cancerous Breast, Mammary Adjacent Tissue, andPlasma. Oxidative medicine and cellular longevity 2015, 6429812, https://doi. org/10.1155/2016/6429812 (2015)

30. Marshall, K. Therapeutic applications of whey protein. Alternative medicine review: a journal of clinical therapeutic 9, 136-156 (2004).

31. Bounous, G. Whey protein concentrate (WPC) and glutathione modulation in cancer treatment. Anticancer Res 20, 4785-4792 (2000).

32. Huang, C. S., Chang, L. S., Anderson, M. E. \& Meister, A. Catalytic and regulatory properties of the heavy subunit of rat kidney gamma-glutamylcysteine synthetase. J Biol Chem 268, 19675-19680 (1993).

33. Suzuki, M., Endo, M., Shinohara, F., Echigo, S. \& Rikiishi, H. Rapamycin suppresses ROS-dependent apoptosis caused by selenomethionine in A549 lung carcinoma cells. Cancer Chemother Pharmacol 67, 1129-1136, https://doi.org/10.1007/s00280-0101417-7 (2011).

34. Chen, W. et al. Synergistic antitumor activity of rapamycin and EF24 via increasing ROS for the treatment of gastric cancer. Redox biology 10, 78-89, https://doi.org/10.1016/j.redox.2016.09.006 (2016).

35. Yellen, P. et al. High-dose rapamycin induces apoptosis in human cancer cells by dissociating mTOR complex 1 and suppressing phosphorylation of 4E-BP1. Cell Cycle 10, 3948-3956, https://doi.org/10.4161/cc.10.22.18124 (2011).

36. Miwa, S. et al. Low abundance of the matrix arm of complex I in mitochondria predicts longevity in mice. Nature communications 5, 3837, https://doi.org/10.1038/ncomms4837 (2014).

37. Glasauer, A. \& Chandel, N. S. Targeting antioxidants for cancer therapy. Biochem Pharmacol 92, 90-101, https://doi.org/10.1016/j. bcp.2014.07.017 (2014).

38. DeNicola, G. M. et al. Oncogene-induced Nrf2 transcription promotes ROS detoxification and tumorigenesis. Nature 475, 106-109, https://doi.org/10.1038/nature10189 (2011).

39. Robida-Stubbs, S. et al. TOR signaling and rapamycin influence longevity by regulating SKN-1/Nrf and DAF-16/FoxO. Cell metabolism 15, 713-724, https://doi.org/10.1016/j.cmet.2012.04.007 (2012)

40. Calap-Quintana, P. et al. TORC1 Inhibition by Rapamycin Promotes Antioxidant Defences in a Drosophila Model of Friedreich's Ataxia. PLoS One 10, e0132376, https://doi.org/10.1371/journal.pone.0132376 (2015).

41. Faes, S., Demartines, N. \& Dormond, O. Resistance to mTORC1 Inhibitors in Cancer Therapy: From Kinase Mutations to Intratumoral Heterogeneity of Kinase Activity. 2017, 1726078, https://doi.org/10.1155/2017/1726078 (2017).

42. Sancak, Y. et al. Ragulator-Rag complex targets mTORC1 to the lysosomal surface and is necessary for its activation by amino acids. Cell 141, 290-303, https://doi.org/10.1016/j.cell.2010.02.024 (2010).

43. Sancak, Y. et al. The Rag GTPases bind raptor and mediate amino acid signaling to mTORC1. Science 320, 1496-1501, https://doi. org/10.1126/science.1157535 (2008).

44. Rojo, A. I., Sagarra, M. R. \& Cuadrado, A. GSK-3beta down-regulates the transcription factor Nrf2 after oxidant damage: relevance to exposure of neuronal cells to oxidative stress. J Neurochem 105, 192-202, https://doi.org/10.1111/j.1471-4159.2007.05124.x (2008).

45. Schreiber, E., Matthias, P., Muller, M. M. \& Schaffner, W. Rapid detection of octamer binding proteins with 'mini-extracts', prepared from a small number of cells. Nucleic acids research 17, 6419 (1989).

\section{Acknowledgements}

This work was supported by grant from the Ministry of Science and Technology, Taiwan (NSC 102-2320-B037018), and Kaohsiung Veterans General Hospital Research Program (VGHKS104-55).

\section{Author Contributions}

Shih-Hsuan Cheng wrote the manuscript, researched the data and contributed to the design of the study. YangMing Tseng and Shih-Meng Tsai researched the data and contributed to the discussion. Szu-Hsien Wu provided technical support. Li-Yu Tsai is the guarantor of this work. All the authors read and approved the final manuscript. 


\section{Additional Information}

Supplementary information accompanies this paper at https://doi.org/10.1038/s41598-017-14159-5.

Competing Interests: The authors declare that they have no competing interests.

Publisher's note: Springer Nature remains neutral with regard to jurisdictional claims in published maps and institutional affiliations.

(c) (i) Open Access This article is licensed under a Creative Commons Attribution 4.0 International License, which permits use, sharing, adaptation, distribution and reproduction in any medium or format, as long as you give appropriate credit to the original author(s) and the source, provide a link to the Creative Commons license, and indicate if changes were made. The images or other third party material in this article are included in the article's Creative Commons license, unless indicated otherwise in a credit line to the material. If material is not included in the article's Creative Commons license and your intended use is not permitted by statutory regulation or exceeds the permitted use, you will need to obtain permission directly from the copyright holder. To view a copy of this license, visit http://creativecommons.org/licenses/by/4.0/.

(C) The Author(s) 2017 